\title{
Discrete Kinetic Eigenmode Spectra of Electron Plasma Oscillations in Weakly Collisional Plasma: A Numerical Study
}

Carrie Black ${ }^{\mathrm{a})}$

Space Weather Laboratory, NASA Goddard Space Flight Center, Greenbelt, MD 20771

Kai Germaschewski

Center for Integrated Computation and Analysis of Reconnection and Turbulence, Institute for the Study of Earth, Oceans, and Space and the Department of Physics, University of New Hampshire Durham, NH 03824

Amitava Bhattacharjee

Center for Integrated Computation and Analysis of Reconnection and Turbulence, Institute for the Study of Earth, Oceans, and Space and the Department of Physics, University of New Hampshire Durham, NH 03824 and

Permanent Address: Princeton Plasma Physics Laboratory, Princeton University,

Princeton, NJ 08543

C.S.Ng

Geophysical Institute, University of Alaska, Fairbanks, Fairbanks, AK, 99775, USA

(Dated: 8 October 2012) 
It has been demonstrated that in the presence of weak collisions, described by the LenardBernstein collision operator, the Landau-damped solutions become true eigenmodes of the system and constitute a complete set [C.-S. Ng, A. Bhattacharjee, and F. N. Skiff, Phys. Rev. Lett. 83, 1974 (1999), C.S. Ng, A. Bhattacharjee, and F. Skiff, Phys. Rev. Lett. 96, 065002 (2004). We present numerical results from an Eulerian Vlasov code that incorporates the Lenard-Bernstein collision operator [A. Lenard and I. B. Bernstein, Phys. Rev. 112, 1456 (1958)]. The effect of collisions on the numerical recursion phenomenon seen in Vlasov codes is discussed. The code is benchmarked against exact linear eigenmode solutions in the presence of weak collisions, and a spectrum of Landau-damped solutions is determined within the limits of numerical resolution. Tests of the orthogonality and the completeness relation are presented.

PACS numbers: 52.20Fs, 52.25.Dg, 52.27.Aj, 52.35.Fp, 52.65.Ff, 52.65.Vv

a)Electronic mail: Carrie.Black@nasa.gov 


\section{INTRODUCTION}

The problem of kinetic damping of plasma waves in a Maxwellian plasma is one of the most fundamental concepts in plasma physics. It is well known that Landau-damped solutions ${ }^{1}$ are not true eigenmodes. The true eigenmodes for a collisionless plasma were obtained by Van Kampen ${ }^{2}$ and $\mathrm{Case}^{3}$. The Case-Van Kampen modes constitute a continuous spectrum, which correspond to the infinite number of degrees of freedom of the system. At this level of description, each particle is considered to move independent of any effects from surrounding particles, and there is no coupling. In most situations of physical interest where the initial conditions are smooth, a broad and continuous spectrum of Case-Van Kampen modes is excited. The Landau-damped solutions emerge, in the long-time limit, as remnants due to the interference of the continuous spectrum of singular Case-Van Kampen eigenmodes.

Lenard and Bernstein $(\mathrm{LB})^{4}$ reconsidered the kinetic problem using an operator of the FokkerPlanck type ${ }^{5}$. They obtained an exact analytic solution with a dispersion relation that formally yields the Landau root in the limit of zero collisions. However, they did not discuss the nature of the spectrum or address the issue of completeness of the eigenmodes in the presence of collisions. In retrospect, this appears a bit surprising because one of the important features of the LenardBernstein collision operator, unlike the Bhatnagar-Gross-Krook (BGK) collision operator ${ }^{6}$, is that, in the limit of zero collision, it leads to a problem in singular perturbation theory in velocity space. While both BGK and LB operators produce the Landau solution in the limit of zero collision, the impact on the spectrum is profoundly different in the case of the LB operator, which arguably is more physical than the BGK operator.

The most recent and direct impetus for theoretical studies on the nature of the kinetic spectrum in the presence of weak collisions have come from the remarkable experiments and analyses of Skiff and co-workers ${ }^{7,8}$, who used laser-induced fluorescence techniques to measure perturbed ion distribution functions in a stable plasma at unprecedented levels of accuracy (by two orders of magnitude compared with previous measurements). They offered the important insight that the linear eigenmode spectrum in the presence of weak collisions is intrinsically discrete. While the Vlasov description smoothens particle discreteness to form a continuous kinetic fluid in phase space and allows particles to move essentially independent of each other except through their role in collectively supporting a self-consistent electric field, binary collisions, embodied in the FokkerPlanck or the LB operator, introduce particle discreteness in the problem, leading to the emergence 
of a discrete spectrum from the continuum modes. While the transformation of continuous spectra to discrete spectra behavior has been known to occur in strongly collisional systems that obey fluid equations (such as hydrodynamics or resistive MHD equations), the weakly collisional kinetic problem has not received the attention it deserves. Following the work of Skiff and co-workers, $\mathrm{Ng}$, Bhattacharjee, and Skiff (NBS) ${ }^{9,10}$, demonstrated that in the presence of weak collisions, the singular Case-Van Kampen continuous spectrum is completely eliminated, and replaced by a discrete and smooth spectrum of eigenmodes which, furthermore, constitute a complete set. The Landau-damped solutions emerge as true eigenmodes of the weakly collisional theory in the limit of zero collision ${ }^{9}$. The demonstration of completeness of the discrete eigenmodes by NBS $^{10}$ appears to resolve a controversy as to whether the total spectrum consists of a discrete as well as a continuous part ${ }^{11}$ or only a discrete spectrum. It should be borne in mind, however, that the actual differentiation of the two types of spectra in real or numerical experiments is a subtle issue.

The main goal of this paper is to test numerically the theoretical results obtained by NBS. For the first time, we report results from a kinetic Eulerian code in $1 \mathrm{~d}-1 \mathrm{v}$ space (that is, phase space consisting of one spatial coordinate $\mathrm{x}$ and one velocity space coordinate $\mathrm{v}$ ) that includes the LB collision operator. We describe the code, hereafter referred to as the Kinetic Code, in Section II. In Section II E, we report on some standard tests of the code and compare the predictions of the code in the presence of collisions with the linear eigenmode analysis of NBS. In Section III, we discuss our effort in testing the completeness relation of NBS eigenmodes, which is a significant numerical challenge. We conclude in Section IV with a summary.

\section{KINETIC CODE}

\section{A. Description of the Numerical Method}

The 1d-1v Kinetic Code integrates the Vlasov-Poisson system, and has been extended to implement the Lenard-Bernstein collision operator in this work:

$$
\begin{array}{r}
\frac{\partial f}{\partial t}+v \frac{\partial f}{\partial x}+\frac{q E}{m} \frac{\partial f}{\partial v}=\nu \frac{\partial}{\partial v}\left[v f+v_{t h}^{2} \frac{\partial f}{\partial v}\right] \\
\nabla \cdot E=\frac{q}{\epsilon_{0}} \int_{-\infty}^{\infty}\left[f(x, v, t)-f_{i}(v, 0)\right] d v
\end{array}
$$

Here $f=f(x, v, t)$ is the electron distribution function, $f_{i}(v, 0)$ is the spatially uniform ion 
background, $m$ is the electron mass, $v_{t h}$ is the electron thermal velocity, $\nu$ is assumed to be a constant collision frequency, $E$ is the self-consistent electric field and $\epsilon_{0}$ is the permittivity of free space.

The system is typically initialized with a Maxwellian distribution, Eq.(3). At $t=0$, this equilibrium is perturbed by a sinusoidal perturbation, Eq.(4)

$$
\begin{array}{r}
f^{(0)}(x, v)=\frac{n_{0}}{\sqrt{2 \pi} v_{t h}} e^{-\frac{v^{2}}{2 v_{t h}^{2}},} \\
f^{(1)}(x, v)=\epsilon \sin (k x) f^{(0)}(x, v),
\end{array}
$$

which corresponds to a perturbed electric field,

$$
E^{(1)}(x)=\epsilon \frac{e n_{0}}{\epsilon_{0} k} \cos (k x)
$$

Here $\epsilon$ is a positive constant, measuring the size of the initial perturbation.

The Kinetic Code employs a numerical method similar to the one proposed by Schumer and Holloway ${ }^{12}$, though it uses a finite difference scheme in space rather than a Fourier spectral method. The spatial boundaries are periodic. The code uses the split-step method of Schumer and Holloway with a 4th order Runge-Kutta method as the time integrator. For the velocity-dependent part of the distribution function we choose the symmetrically weighted Hermite functions as a spectral basis, defined as

$$
\psi_{n}(v)=\psi^{n}(v)=C_{n} e^{-v^{2} / 2} H_{n}(v)
$$

where $H_{n}(v)$ is the $n^{t h}$ Hermite polynomial and $C_{n}=\pi^{-1 / 4}\left(2^{n} n !\right)^{-1 / 2}$ is a normalization constant. These functions are orthonormal and satisfy the following recursion relations:

$$
\begin{array}{r}
v \psi^{n}(v)=\sqrt{\frac{n+1}{2}} \psi^{n+1}+\sqrt{\frac{n}{2}} \psi^{n-1}(v), \\
\frac{d}{d v} \psi^{n}(v)=-\sqrt{\frac{n+1}{2}} \psi^{n+1}+\sqrt{\frac{n}{2}} \psi^{n-1}(v) .
\end{array}
$$

Following Schumer and Holloway ${ }^{12}$, these recursion relations are used to numerically implement the 1.h.s. of Eq. (1). The spatial derivative is generally implemented using 2nd order central difference and the Poisson equation is solved by inverting a tri-diagonal matrix. However, for the simulations in this work, we used the linear, single-mode option of the code, which implements spatial derivatives exactly by multiplication with $i k$. 


\section{B. Implementation of the Lenard-Bernstein Collision Operator}

The Lenard-Bernstein collision operator ${ }^{4}$ operator comes from the full Fokker-Planck operator by linearizing and then assuming constant collision frequency, and single spatial and velocity dimensions. Its effect is to relax a non-thermal distribution function to a Maxwellian with a prescribed thermal velocity. It is known to conserve particle number and, for non-drifting distribution functions, momentum. These collisions have been implemented in the Kinetic Code by exploiting the recursion relations, Eqs. (7) and(8).

Given a representation of the distribution function expanded in symmetrically weighted Hermite functions, and leaving out the spatial and temporal dependence for brevity,

$$
f(v)=\sum_{n=0}^{\infty} f_{m} \psi_{n}(v),
$$

the right hand side of Eq. (1) is expanded and Eqs.(7), (8) are applied.

$$
\begin{aligned}
& L B \equiv \nu \frac{\partial}{\partial v}\left[v f+v_{t h}^{2} \frac{\partial f}{\partial v}\right], \\
&=\nu \sum_{n=0}^{\infty} f_{m} \frac{\partial}{\partial v}\left[\sqrt{\frac{n+1}{2}}\left(1-v_{t h}^{2}\right) \psi_{n+1}+\sqrt{\frac{n}{2}}\left(1+v_{t h}^{2}\right) \psi_{n-1}\right], \\
&=\nu \sum_{n=0}^{\infty} f_{m}\left[-\frac{\sqrt{(n+1)(n+2)}}{2}\left(1-v_{t h}^{2}\right) \psi_{n+2}\right. \\
&+\left(\frac{n+1}{2}\left(1-v_{t h}^{2}\right)-\frac{n}{2}\left(1+v_{t h}^{2}\right)\right) \psi_{n} \\
&\left.+\frac{\sqrt{n(n-1)}}{2}\left(1+v_{t h}^{2}\right) \psi_{n-2}\right] .
\end{aligned}
$$

Expanding the 1.h.s. as well,

$$
L B=\sum_{n=0}^{\infty} L B_{n} \psi_{n}(v),
$$

and matching coefficients on both sides, we find the algorithm implemented in the code:

$$
\begin{aligned}
L B_{n}=\nu[ & -\frac{\sqrt{(n-1) n}}{2}\left(1-v_{t h}^{2}\right) \\
& +\left(\frac{1}{2}-\frac{2 n+1}{2} v_{t h}^{2}\right) \\
& \left.+\frac{\sqrt{(n+1)(n+2)}}{2}\left(1+v_{t h}^{2}\right)\right] .
\end{aligned}
$$




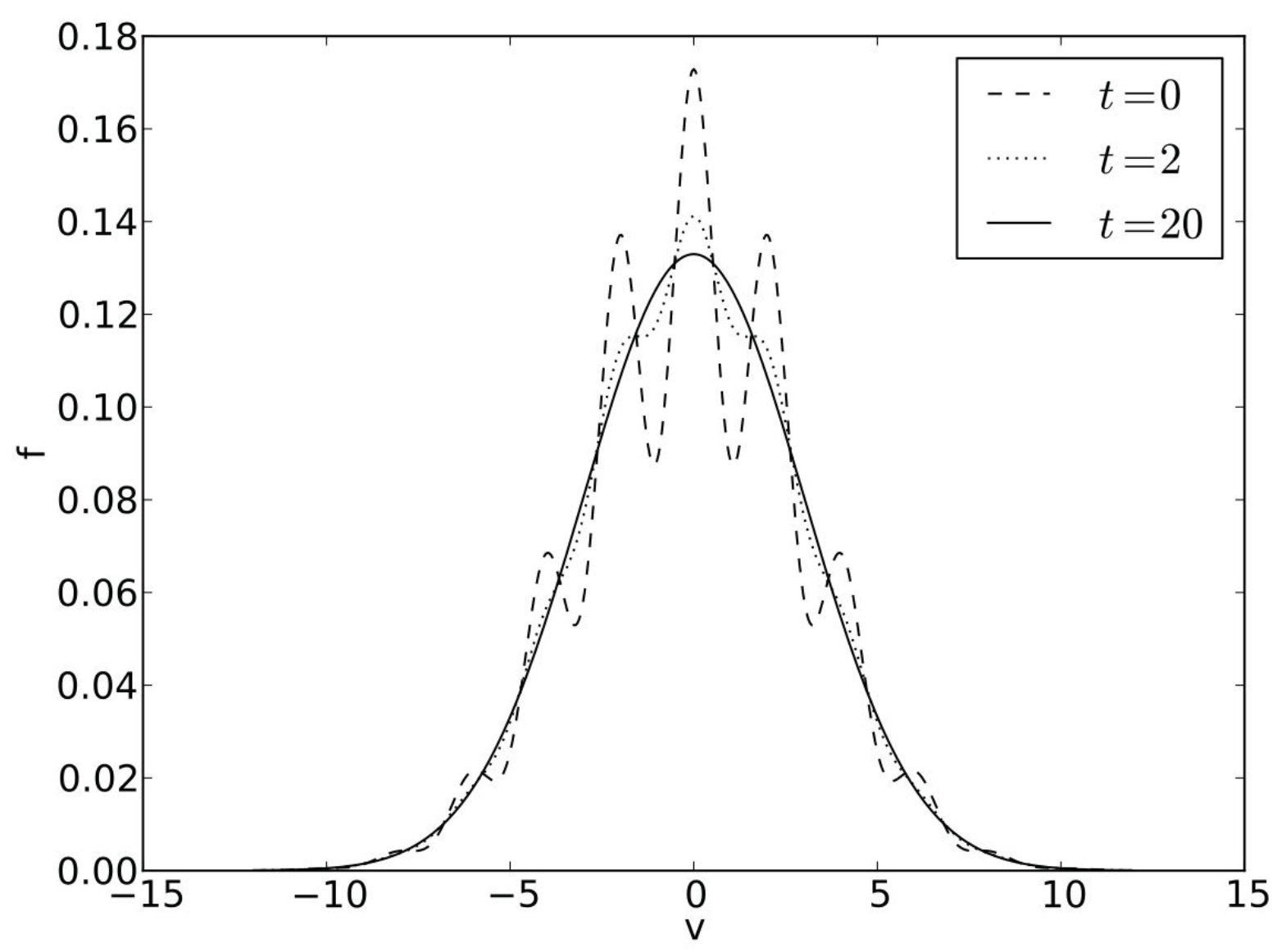

FIG. 1. Evolution of non-Maxwellian distribution function in the collisional Vlasov-Poisson system. Shown is the distribution function $f(v)$ as a function of velocity at the initial time $t=0$ (dashed), at an intermediate time $t=2$ (dotted) where the oscillations have already strongly damped, and at $t=20$ (solid), where the system has fully relaxed to a Maxwellian distribution.

\section{LB Operator Test: Thermalization of a Non-thermal Distribution}

The LB collision operator thermalizes non-Maxwellian distribution functions. Here we demonstrate how our implementation in the Kinetic Code exhibits this behavior.

We start with a filamented perturbed Maxwellian initial condition for the distribution function $f=f(v, t)$ :

$$
f(v, 0)=(1+\epsilon \cos K v) \frac{1}{\sqrt{2 \pi} v_{t h}} e^{-\frac{v^{2}}{2 v_{t h}^{2}}} .
$$

Figure 1 shows the temporal evolution of the distribution function computed by the Kinetic Code, where we chose $\epsilon=0.3, K=3, v_{t h}=3$ and $\nu=0.01$. It can be clearly seen how the 
oscillatory perturbation gets rapidly damped and the system evolves to a Maxwellian distribution with width $v_{t h}$.

\section{Effects of collisions on numerical recursion}

Eulerian Vlasov solvers are limited numerically by velocity-space filamentation of the distribution function. In the absence of collisions, velocity shearing in phase space occurs inevitably due to the free-streaming or ballistic motion of the particles, represented by the first two terms in the left-hand-side of Eq. (1) ${ }^{13-16}$.

This standard phenomenon is illustrated in Fig. 2. We begin at (a) $t=0$ showing the initial perturbation in the distribution function $f_{1}$ (see Eq. (4)). A little while later, in (b), particles with large velocities will have moved further in space than those with smaller velocities. At a later time in (c), the distribution function consists of fine filaments in velocity space. In the collisionless Vlasov-Poisson system, there is no mechanism to suppress the increasingly fine filamentation, so eventually the velocity scales fall below the grid size, leading to the well-known phenomenon of numerical recursion. In the context of the present method, one can postpone this time by increasing resolution or, to some extent, by rescaling the velocity space basis function, as discussed at length by Schumer and Holloway ${ }^{12}$.

Figure 3 demonstrates the consequence of numerical recursion: The electric field in the collisionless run (thin line) follows the exponentially damped standing wave correctly initially, but recursion occurs at $t \approx 150$ where the simulated electric field shows erroneous behavior. The same plot demonstrates that a small collisionality of $\nu=10^{-4}$ is sufficient to avoid numerical recursion, so that the exponential decay continues. It should be noted that while the initial evolution is the same in both cases, in the collisional case the least damped collisional eigenmode eventually dominates as other modes in the initial condition decay away, which, by virtue of being an eigenmode, does not exhibit any further steepening of the velocity space gradients. Figure 4 shows the structure of this eigenmode.

To more closely investigate the occurence of recursion in Vlasov solvers and its suppression by the Lenard-Bernstein collision operator, it is instructive to consider the free-streaming approximation to the Vlasov equation:

$$
\frac{\partial f}{\partial t}+v \frac{\partial f}{\partial x}=\nu \frac{\partial}{\partial v}\left[v f+v_{t h}^{2} \frac{\partial f}{\partial v}\right]
$$



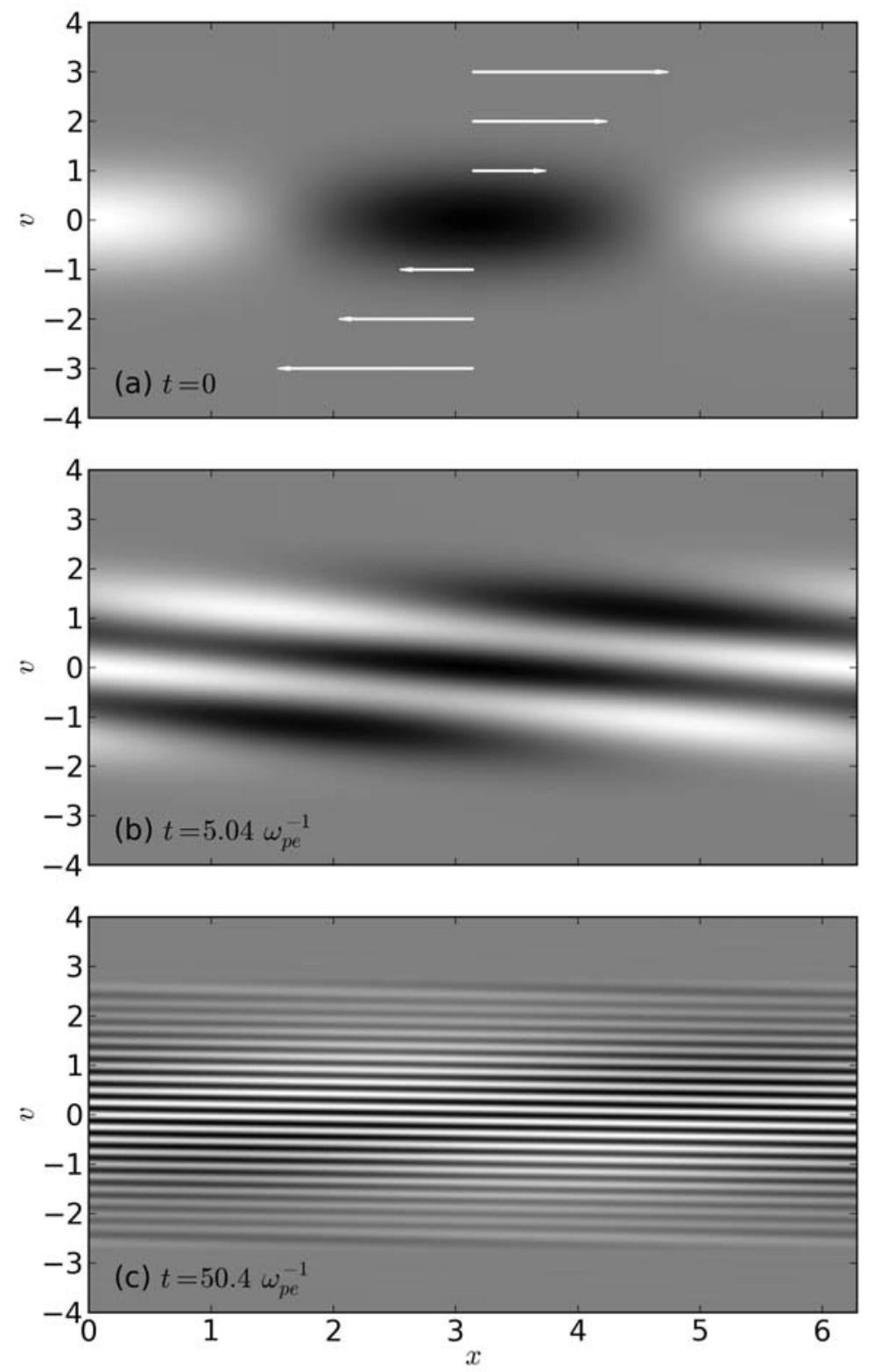

FIG. 2. Evolution of the perturbed distribution function $f_{1}$. As indicated in (a) by the arrows, different parts of the distribution function will move at their own velocity, leading to the distribution function being sheared and formation of filaments as shown at later times in (b) and (c).

Setting the collision frequency $\nu$ to zero for the moment, the equation is easily integrated along its characteristic, and in particular for a typical perturbation with wave number $k$ in position space and Maxwellian distribution in velocity space $f(x, v, 0)=a_{k} \cos (k x) \exp \left(-v^{2} /\left(2 v_{t h}^{2}\right)\right)$, we find 


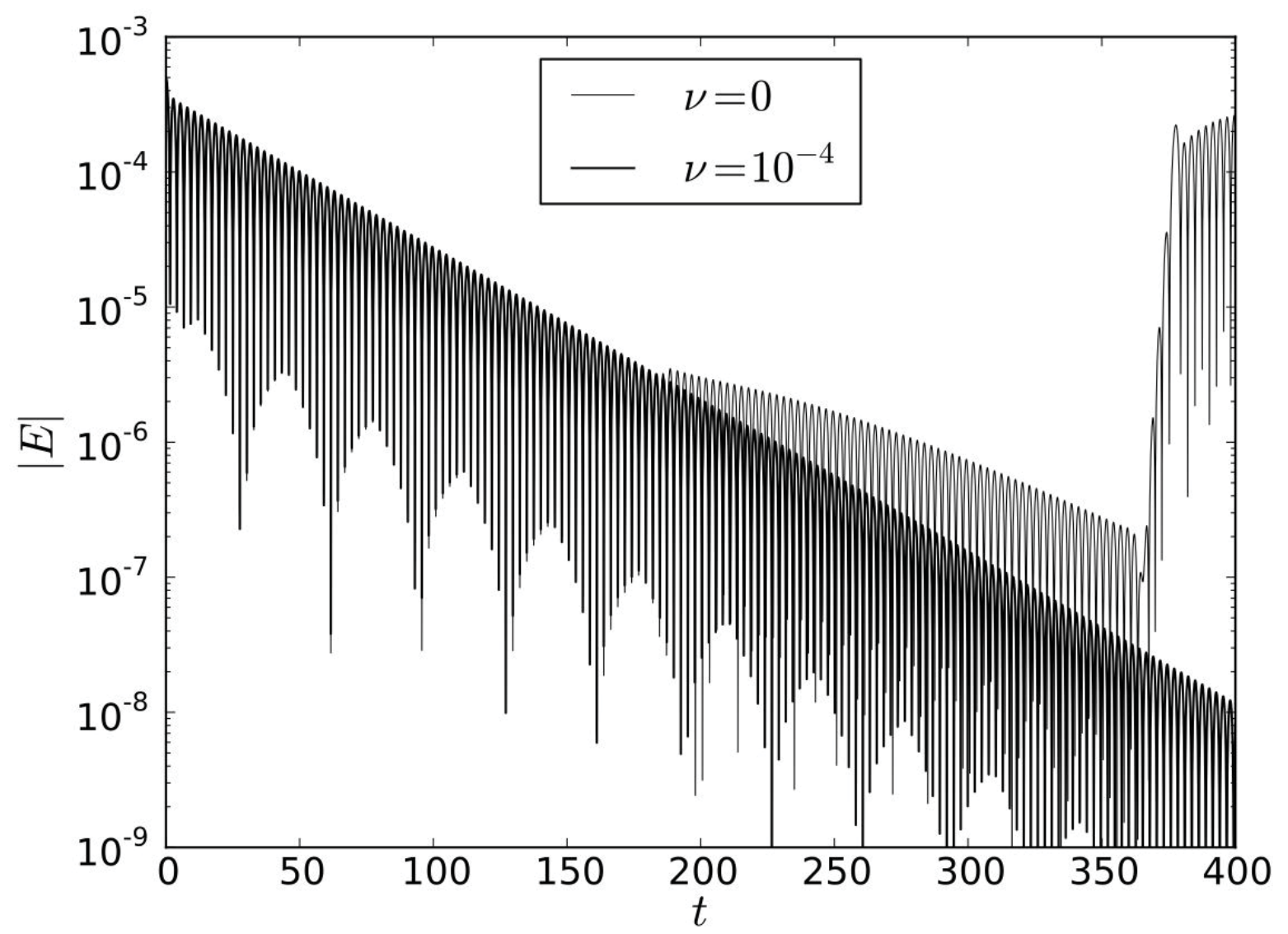

FIG. 3. Evolution of electric field in the collisionless case $(\nu=0$, light line), showing numerical recursion at $t \approx 180 \omega_{p e}^{-1}$ and suppression of the recursion by collisionality $\nu=10^{-4}$ (thick line).

the solution:

$$
f(x, v, t)=f(x-v t, v, 0)=a_{k} \cos k(x-v t) e^{-\frac{v^{2}}{2 v_{t h}^{2}}} .
$$

Taking a cut in velocity space at an arbitrary location $x_{0}$, we see that the distribution function now has an oscillatory dependence $\propto \cos (k t v)$ on $v$ in addition the Maxwellian envelope, similar to our test initial condition in Fig. 1. The period in $v$-space, $2 \pi /(k t)$ decreases in time, and it is clear that the oscillation will become unresolved as we resolve a single cosine period with less than 4 points in $v$ space:

$$
\frac{2 \pi}{k \tau_{\text {recur }}}=4 \Delta v \Longrightarrow \tau_{\text {recur }}=\frac{\pi}{2 k \Delta v}
$$

As the width of Hermite functions is approximately the square root of their order, the resolution of our velocity-scaled basis functions is $\Delta v \approx \frac{U \sqrt{N_{u}}}{N_{u}}$, hence we obtain the recursion time as Schumer 


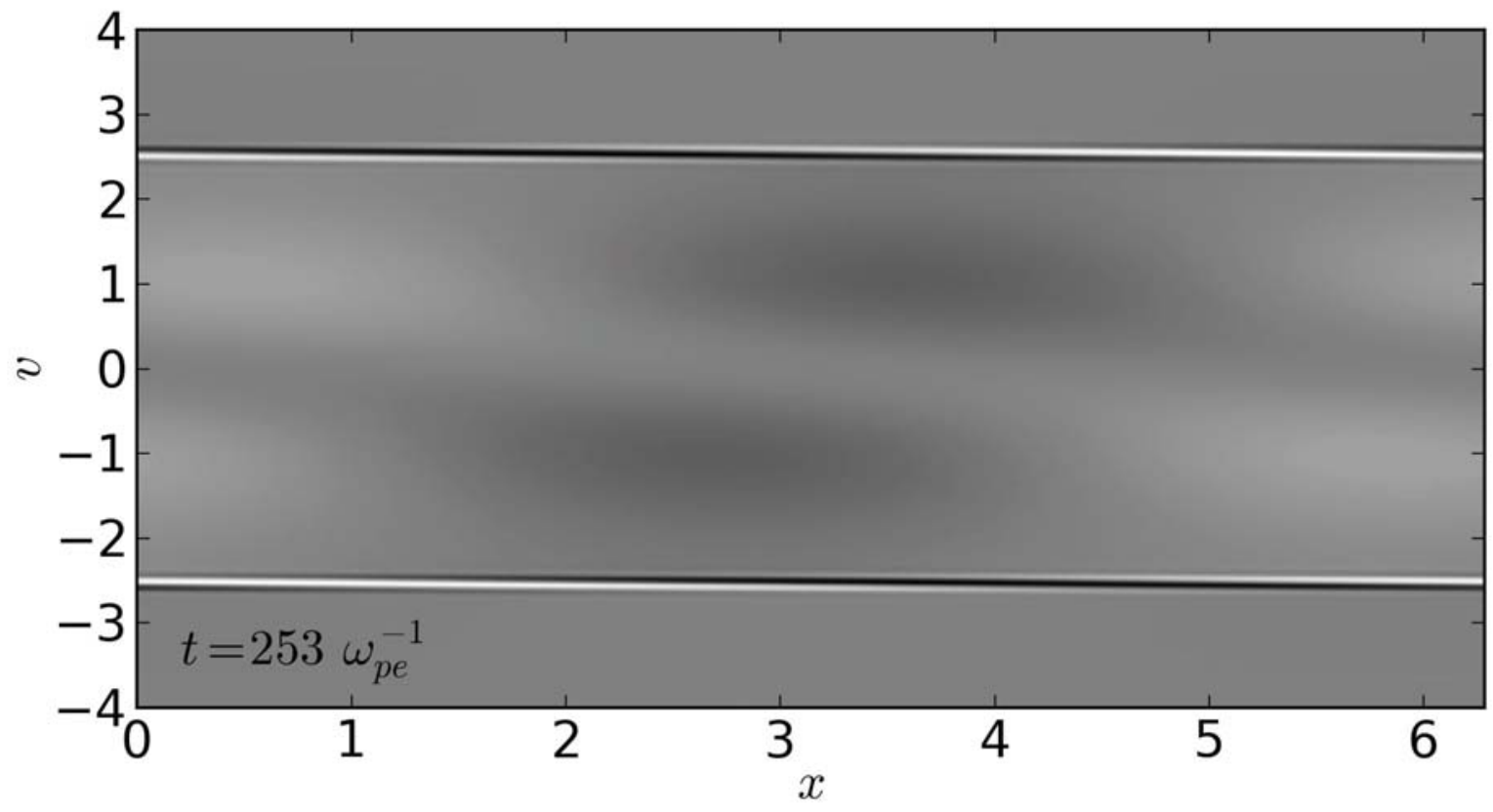

FIG. 4. Structure of the late-time perturbed distribution function $f_{1}$ in the collisional case, $\nu=10^{-4}$. The initial evolution is virtually identical the the collisionless evolution shown in Fig. 2, but at late time (shown is $\left.t=253 \omega_{p e}^{-1}\right)$, the system has evolved into the least-damped collisional eigenmode.

and Holloway ${ }^{12}$ :

$$
\tau_{\text {recur }} \approx \frac{\pi \sqrt{N_{u}}}{2 k U}
$$

We performed a number of runs varying the velocity space resolution $N_{u}$ from 64 through 2048 and determined the recursion times. Figure 5 confirms the expected $N_{u}^{1 / 2}$ scaling of the recursion time.

While recursion at a finite time is unavoidable for numerical solutions of collisionless VlasovPoisson system, $\mathrm{Ng}$ et al. $^{9}$ showed that the character of the solutions changes fundamentally in the presence of collisions. In particular, the Landau damped solutions are transformed into actual eigenmodes, which as such remain unchanged during the temporal evolution, instead of becoming more and more oscillatory in velocity space. It is therefore expected that the numerical solver can follow the time evolution for long times, the phenomenon of recursion should disappear given sufficient resolution.

Turning back to Eq.(14), the initial evolution of the system at finite but small collisionality $\mu$ will be virtually identical, since the initial Maxwellian profile does not have large gradients, so 


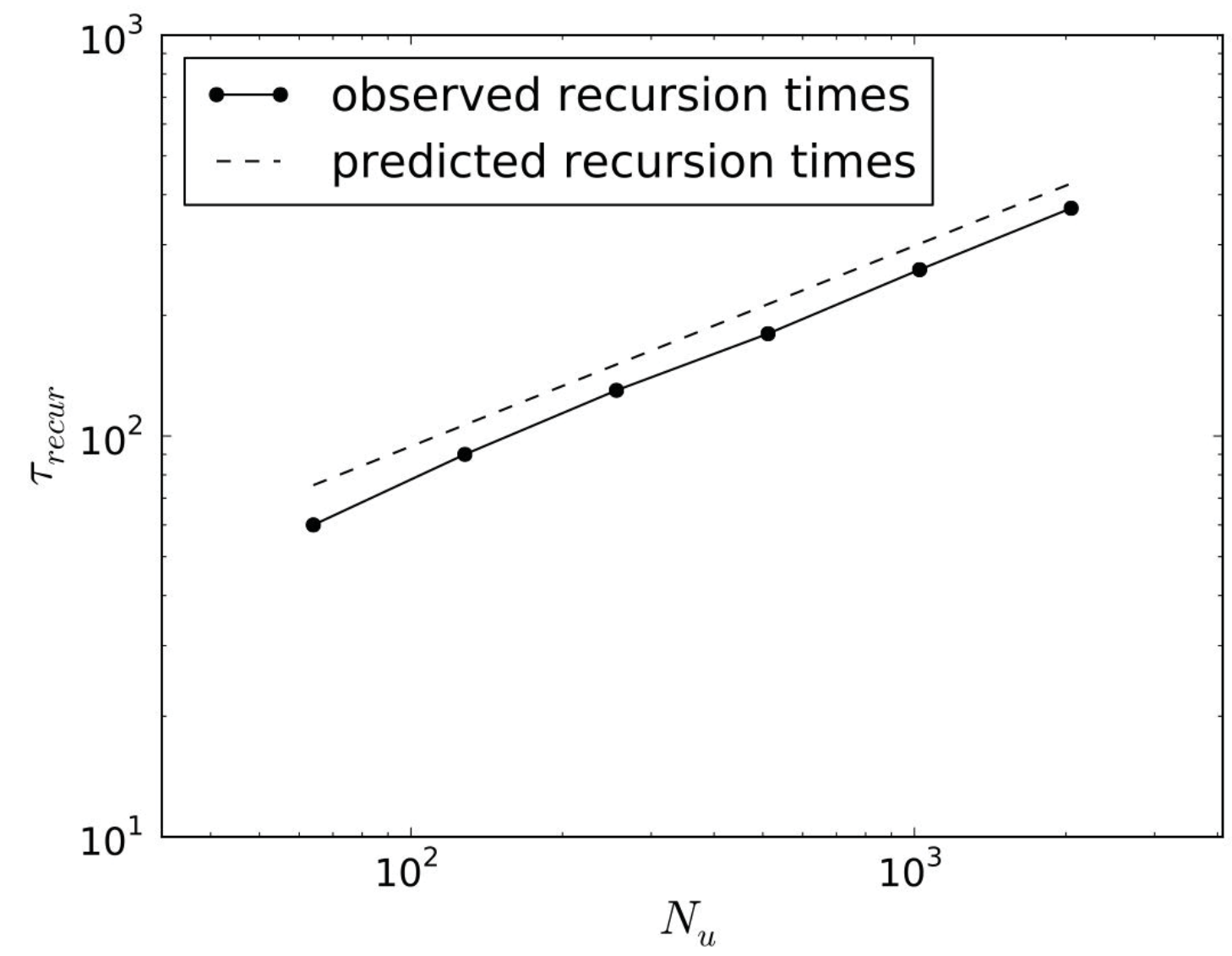

FIG. 5. Numerical recursion time $\tau_{\text {recur }}$ as a function of velocity space resolution $N_{u}$. The observed recursion times compare well to the model $\tau_{\text {recur }} \approx \pi \sqrt{N_{u}} /(2 k U)$. Parameters were $k=1, v_{t h}=1 / 3, U=$ $.5 v_{t h}$.

the collisional terms on the right hand side are small. As time goes on, we again get the filamentation, leading to growing gradients in velocity space. Eventually, the collisional terms becomes important and serves to suppress growth of even higher modes, the dissipative term $\nu v_{t h}^{2} \partial^{2} f / \partial v^{2}$ making the most important contribution. Substituting in our previously obtained free-streaming solution for $f$ (Eq. (15)) and postulating that the collisional term quenches further filamentation once its contribution is comparable to the decay rate $|\gamma|$, we find

$$
\tau_{\text {coll }} \approx \sqrt{\frac{|\gamma|}{\nu}} \frac{1}{\left(k v_{t h}\right)^{2}}
$$

Recursion is prevented if further filamentation is suppressed before the recursion time, ie., $\tau_{\text {coll }}<\tau_{\text {recur }}$. To test this criterion, we solve for the minimum collision frequency needed to 


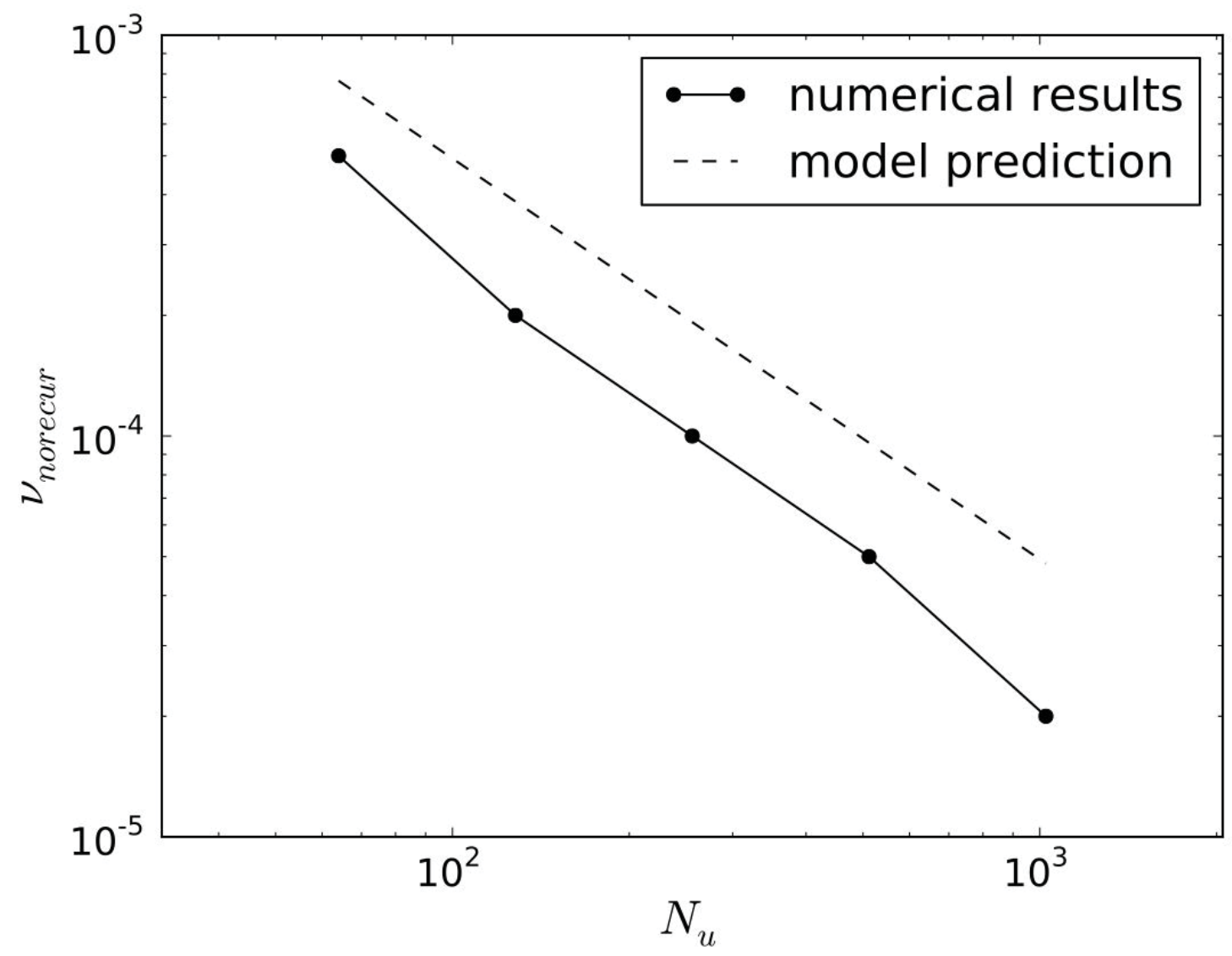

FIG. 6. Collisionality $\nu_{n o r e c u r}$ required to prevent recursion as a function of velocity space resolution $N_{u}$. Parameters were $k=1, v_{t h}=1 / 3, U=.5 v_{t h}$.

prevent recursion given a velocity space resolution $N_{u}$ :

$$
\nu_{\text {norecur }} \approx \frac{(2 k U)^{2} \gamma}{\pi^{2}\left(k v_{t h}\right)^{4} N_{u}} .
$$

Figure 6 shows the collision frequency $\nu$ needed to avoid the recursion previously observed in the collisionless system. It confirms the expected $N_{u}^{-1}$ scaling.

\section{E. Kinetic Code Verification}

We have benchmarked the collisionless version of the Kinetic Code with roots of the plasma dispersion relation from a solver written by C.-S. Ng (referred to hereafter as the Plasma Dispersion Function (PDF) Solver). The relevant dispersion equation is:

$$
1+\alpha(1+\Omega Z(\Omega))=0
$$




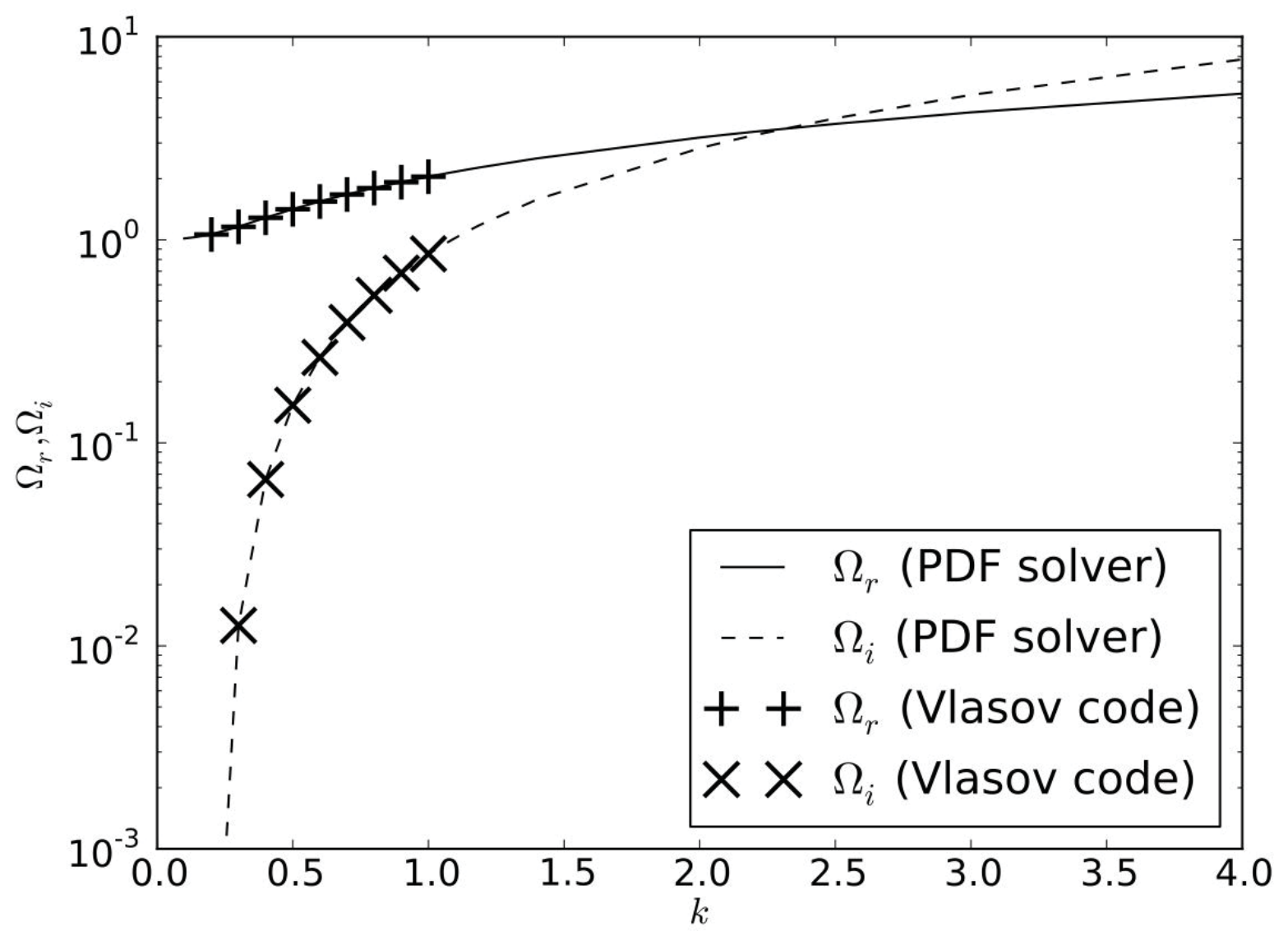

FIG. 7. The Langmuir Dispersion Relation. The upper plots show the frequency of oscillation normalized by plasma frequency. The lower plot shows the damping rate normalized by plasma frequency. "PDF root" is the roots from the Plasma Dispersion Function Solver. "Code root" is the result from the Kinetic Code.

where $\alpha=\omega_{p}^{2} /\left(k v_{t h}\right)^{2}, \Omega=\omega /\left(\sqrt{2} k v_{t h}\right)$, and $Z(\Omega)$ is the plasma dispersion function. As shown in Fig. 7, very good agreement is found between the Kinetic Code and the PDF Solver.

We have also performed benchmarks of the damping of collisional Langmuir waves with the Kinetic Code. Table II, show that the damping rate matches the NBS eigensolver predictions. In the long-wavelength approximation, the eigenfrequencies, $\Omega$, take the form

$$
\Omega=\Omega_{0}+\Omega_{1} \mu
$$

where,

$$
\Omega_{1}=\frac{i \Omega_{0}^{2}\left[\frac{1}{3 \alpha}-\left(\frac{1}{3}+\frac{1}{\alpha}\right) \frac{1}{2 \Omega_{0}^{2}}\right]}{-\frac{1}{\alpha}+\left(1+\frac{1}{\alpha}\right) \frac{1}{2 \Omega_{0}^{2}}} .
$$


TABLE I. NBS modesolver damping rates compared with the correction formulae, Eqs. (21) and (22).

\begin{tabular}{ccccc}
\hline \hline$\alpha$ & $\mu$ & $\Omega_{i}$ NBS & $\Omega_{i}$ Correction Formula & Relative Error \\
\hline 4.0 & 0.01 & -0.22254755 & -0.22253905 & $0.96565 * 10^{-5}$ \\
5.0 & 0.01 & -0.17044662 & -0.17044252 & $0.97423 * 10^{-5}$ \\
9.0 & 0.1 & -0.12701015 & -0.12863040 & $0.96470 * 10^{-3}$ \\
20.0 & 0.1 & -0.06674260 & -0.68091868 & $0.55108 * 10^{-3}$ \\
25.0 & 0.1 & -0.06149031 & -0.61999834 & $0.35246 * 10^{-3}$
\end{tabular}

TABLE II. NBS Benchmark of Kinetic Code. Damping rates for various collision frequencies, $\mu$, recovered from fitting simulation results, compared to those predicted by the NBS eigenmode solver.

\begin{tabular}{lccc}
\hline \hline$\mu$ & $\Omega_{i}$ NBS & $\Omega_{i}$ Kinetic Code Relative Error \\
\hline 0 & -0.0548864 & -0.0549166 & $5.50 \times 10^{-4}$ \\
0.00001 & -0.0548937 & -0.0549240 & $5.52 \times 10^{-4}$ \\
0.0001 & -0.0549601 & -0.0549904 & $5.51 \times 10^{-4}$ \\
0.001 & -0.0556237 & -0.0556539 & $5.43 \times 10^{-4}$ \\
0.01 & -0.0622458 & -0.0622758 & $4.82 \times 10^{-4}$ \\
0.1 & -0.1270101 & -0.1269715 & $3.04 \times 10^{-4}$ \\
\hline \hline
\end{tabular}

Table I shows the comparison between the numerical results obtained by NBS, and the correction formulae (21) and (22).

\section{NBS EIGENMODE STUDY}

This section contains tests of the NBS eigenmodes. First, orthogonality of the modes is examined analytically and numerically. Next, we show that the NBS eigenmodes behave in the collisional Vlasov-Poisson system as predicted. Finally, we present some evidence that are not consistent with the property of completeness. (For numerical reasons, discussed below, we are not able to test the completeness property definitively.) 


\section{A. Orthogonality}

NBS $^{9}$ renormalized and Fourier-transformed the distribution function $f$ in space and time, and recast the collisional Vlasov-Poisson system, Eqs. (1), in terms of a normalized distribution function $g(v)$ :

$$
(u-\Omega) g-\eta \int_{-\infty}^{\infty} g\left(u^{\prime}\right) d u^{\prime}=-i \mu \frac{d}{d u}\left(u g+\frac{1}{2} \frac{d g}{d u}\right)
$$

where $u \equiv v /\left(\sqrt{2} v_{0}\right), g(u) \equiv \sqrt{2} v_{0} f / n_{0}, g_{0} \equiv \exp \left(-u^{2}\right) / \sqrt{\pi}, n_{0}$ is the equilibrium electron density, $\eta(u) \equiv \alpha / 2 d g_{0} / d u$, and $\mu \equiv \nu /\left(\sqrt{2} k v_{0}\right)$. This is an eigenmode equation with solutions $g_{m}$ belonging to complex eigenfrequencies $\Omega_{m}$. NBS found the corresponding adjoint equation and showed that its adjoint solutions $G_{m}$ are related to the eigenfunctions $g_{m}$ by

$$
G_{n}(u) \propto g_{n}(u) e^{u^{2}}+\frac{\alpha}{\sqrt{\pi}} \int_{-\infty}^{\infty} g_{n}\left(u^{\prime}\right) d u^{\prime}
$$

By construction, $G_{n}$ and $g_{m}$ belonging to different eigenfrequencies $\Omega_{n}, \Omega_{m}$ are bi-orthogonal, that is,

$$
\int_{-\infty}^{\infty} G_{n}(u) g_{m}(u) d u=0
$$

This condition allows us to determine the expansion coefficients $c_{n}$ for expanding an arbitrary function $g(u)=\sum_{m=0}^{\infty} c_{m} g_{m}(u)$ in terms of eigenfunctions $g_{m}$, after the $G_{m}$ are appropriately normalized:

$$
c_{m}=\int_{-\infty}^{\infty} G_{m}(u) g(u) d u
$$

We have confirmed numerically bi-orthogonality for a number of eigenfunctions obtained by the method described in ${ }^{9}$. The eigenfunctions are provided as a set of Hermite expansion coefficients $\left\{a_{m n}\right\}$ such that

$$
g_{m}(v)=\sum_{n=0}^{\infty} a_{m n} C_{n} H_{n}(v) e^{-v^{2}}
$$

where $C_{n}=1 /\left(\pi^{1 / 4} \sqrt{2^{k} k !}\right)$. We express the adjoint function $G_{m}$ in terms of Hermite polynomials, too, but without the weight function $e^{-v^{2}}$ to enable us to use the orthogonality property of Hermite polynomials to evaluate integrals. We write:

$$
G_{m}(v)=\sum_{n=0}^{\infty} A_{m n} C_{n} H_{n}(v) .
$$


TABLE III. Numerical confirmation of the orthonormality of the NBS eigenmodes. Shown is $\int G_{n} g_{m} d u$ for Least Damped Mode and Modes 1 to 4 for $\mu=0.1$

\begin{tabular}{|c|c|c|c|c|c|}
\hline$n \backslash m$ & LDM & 1 & 2 & 3 & 4 \\
\hline LDM & 1 & $6.6788 \mathrm{e}-14$ & $2.4654 \mathrm{e}-10$ & $4.3470 \mathrm{e}-07$ & $3.3390 \mathrm{e}-05$ \\
\hline 1 & $1.0726 \mathrm{e}-14$ & & $1.9583 \mathrm{e}-09$ & $2.5373 \mathrm{e}-06$ & $1.6947 \mathrm{e}-04$ \\
\hline 2 & $2.1735 \mathrm{e}-11$ & $1.0749 \mathrm{e}-09$ & 1 & $6.8720 \mathrm{e}-06$ & $3.4759 \mathrm{e}-04$ \\
\hline 3 & $2.6671 \mathrm{e}-08$ & $9.6933 \mathrm{e}-07$ & $4.7828 \mathrm{e}-06$ & & 7.6067e-04 \\
\hline 4 & $1.5850 \mathrm{e}-06$ & $5.0094 \mathrm{e}-05$ & $1.8717 \mathrm{e}-04$ & $5.8853 \mathrm{e}-04$ & 1 \\
\hline
\end{tabular}

Substituting the expansions for $G_{n}, g_{n}$ into Eq. (24), multiplying by $C_{m} H_{m}(u) \exp \left(-u^{2}\right)$ and integrating over $u$ in the usual manner, we determine the coefficients $A_{n m}$ :

$$
A_{n m}=b_{n}\left[a_{n m}+\alpha a_{n 0} \delta_{m 0}\right]
$$

since $\int_{-\infty}^{\infty} g_{n}(u) d u=\pi^{1 / 4} a_{n 0}$. The $b_{n}$ are yet undetermined normalization constants. For any function $g(u)$ expanded in Hermite functions, $g(u)=\sum_{n=0}^{\infty} a_{n} C_{n} H_{n}(u) \exp \left(-u^{2}\right)$, we can perform a change of basis to find its coefficients $\left\{c_{m}\right\}$ for an expansion in collisional eigenfunctions:

$$
\begin{aligned}
c_{m} & =\int_{-\infty}^{\infty} G_{n}(u) g(u) d u, \\
& =\sum_{k=0}^{\infty} A_{n k} a_{k}=b_{n}\left[(1+\alpha) a_{n 0} a_{0}+\sum_{k=1}^{\infty} a_{n k} a_{k}\right] .
\end{aligned}
$$

The normalization coefficients can now be determined by plugging in $g_{n}$ for $g$, for which we require

$$
\begin{aligned}
& 1=\int_{-\infty}^{\infty} G_{n} g_{n} d u,=b_{n}\left[(1+\alpha) a_{n 0}^{2}+\sum_{k=1}^{\infty} a_{n k}^{2}\right] \\
& \Longrightarrow b_{n}=\left[(1+\alpha) a_{n 0}^{2}+\sum_{k=1}^{\infty} a_{n k}^{2}\right]^{-1} .
\end{aligned}
$$

Table III shows an example calculation where we have calculated and normalized the adjoint functions $G_{n}$ and checked that the resulting functions are in fact bi-orthogonal. It is evident that the numerical eigenfunctions exhibit large and rapid oscillations near the phase speed of the wave, which makes it numerically challenging to determine them to very high accuracy. 


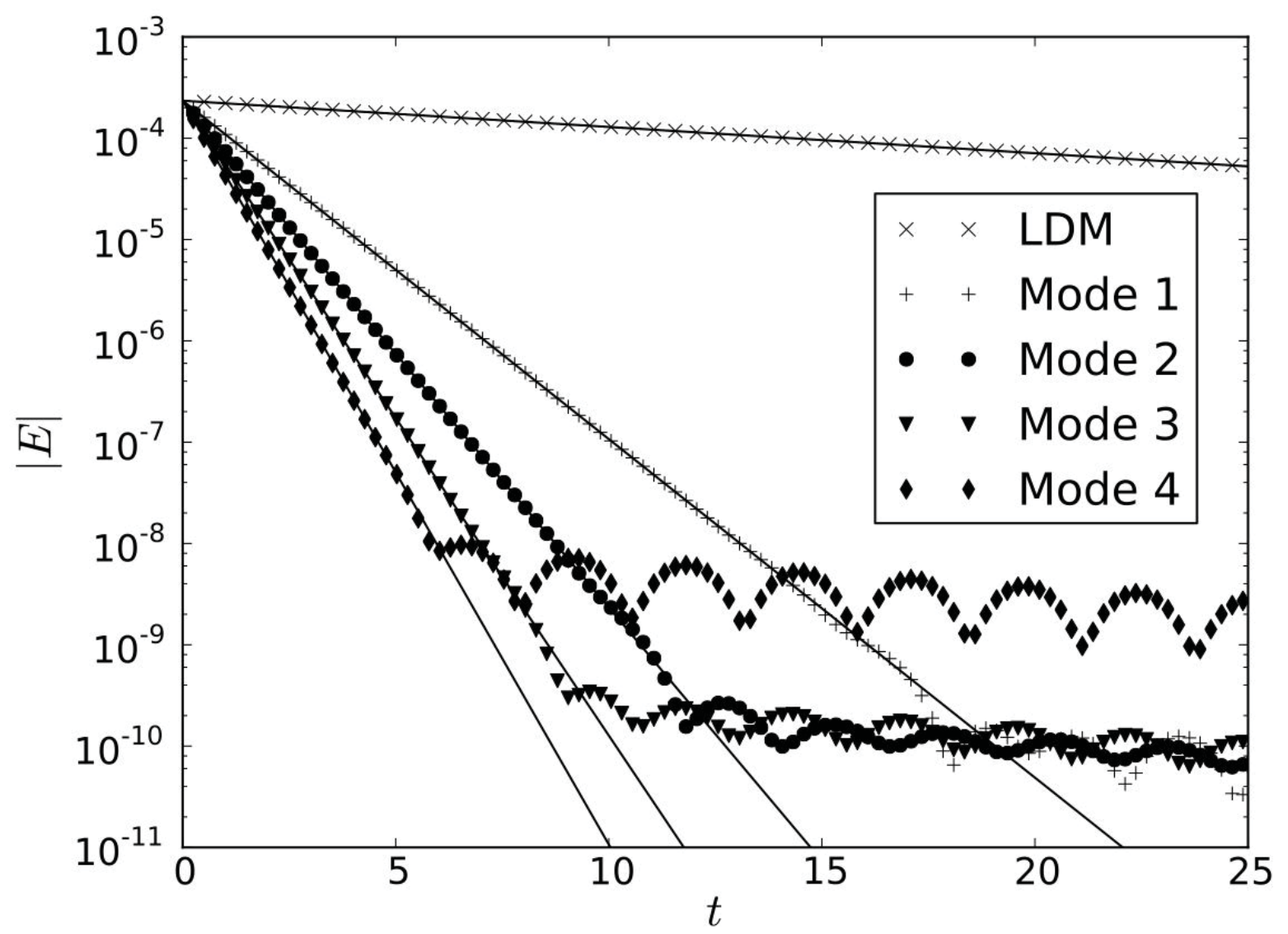

FIG. 8. Time evolution of the $k=1$ component of the electric field for separate simulations initialized with LDM and Modes 1 to $4, \mu=0.1$. Solid lines show the best fit to the exponential decay phase.

\section{B. Structure of the Eigenmodes}

The NBS eigensolver identified the least damped mode and 4 further modes for $\mu=0.1$. The complex eigenfrequencies are given in Table IV.

Figure 8 shows the time evolution of Vlasov simulations initialized with eigenmodes found by the NBS eigensolver for $\mu=0.1$. Indicated are the electric field (symbols) and least square fits to the exponential decay part of the evolution. It can be clearly seen that all modes initially show exponential decay as expected for an eigenmode, and examination of the full distribution function output confirmed this, as well. For all modes other than the least damped mode (LDM), the exponential decay eventually breaks down and we see further slow decay at the growth rate of the LDM.

We compare the growth rates found from the Vlasov simulations to the imaginary part of $\Omega$ 
TABLE IV. Growth rates of the collisional eigenmodes at $\mu=0.1$, showing that the results of the Vlasov code agree very well with the eigenvalues found by the NBS solver.

\begin{tabular}{|c|c|c|c|}
\hline Mode & $\Omega_{i}$ NBS & $\Omega_{i}$ Vlasov code & Relative Error \\
\hline LDM & I - -0.12701014 & -0.12700989 & $1.97 \times 10^{-6}$ \\
\hline 1 & -1.63253445 & -1.63253647 & $1.24 \times 10^{-6}$ \\
\hline 2 & -2.44408929 & -2.44409574 & $2.64 \times 10^{-6}$ \\
\hline 3 & -3.06241689 & -3.06242860 & $3.83 \times 10^{-6}$ \\
\hline 4 & -3.58772051 & -3.58797131 & $6.99 \times 10^{-5}$ \\
\hline
\end{tabular}

calculated by the eigensolver in Table. IV. The Vlasov code clearly captures the temporal evolution of the eigenmodes to high accuracy in the initial phase of the simulations.

The reason why the higher modes seemingly cease to evolve according to their linear dynamics can be understood better by considering the shape of the eigenfunctions in velocity space, as plotted in Fig. 9. All the eigenfunctions $g_{m}$ are normalized with respect to their zeroth moment, ie., $\int_{-\infty}^{\infty} g_{m}(v) d v=1$, hence they all contribute equally to the charge density and also the electric field. In particular, while all the modes in Fig. 8 start at the same value of the electric field, the distribution function $g_{4}$ of Mode 4 is actually up to $10^{6}$ times larger than the distribution function of the least damped mode $g_{L D M}$. Due to numerical discretization error, the initial Mode 4 is not exact, but contains small contributions from other modes, in particular the least damped mode. An LDM error component of $10^{-6}$ of the magnitude of Mode 4 would create an error in the electric field of order unity already. While the initial LDM error component is actually smaller, about $10^{-10}$, it decays away much slower than the main mode, so eventually the electric field contributed by this small component overwhelms the electric field of the main mode and shows up as the slowly decaying evolution after the initial exponential phase. It should be noted that the structure of the eigenmodes is numerically rather challenging - they contain large oscillatory components that almost cancel out as one integrates over $v$-space, which leads to a significant loss of precision when performed in floating point arithmetic. 


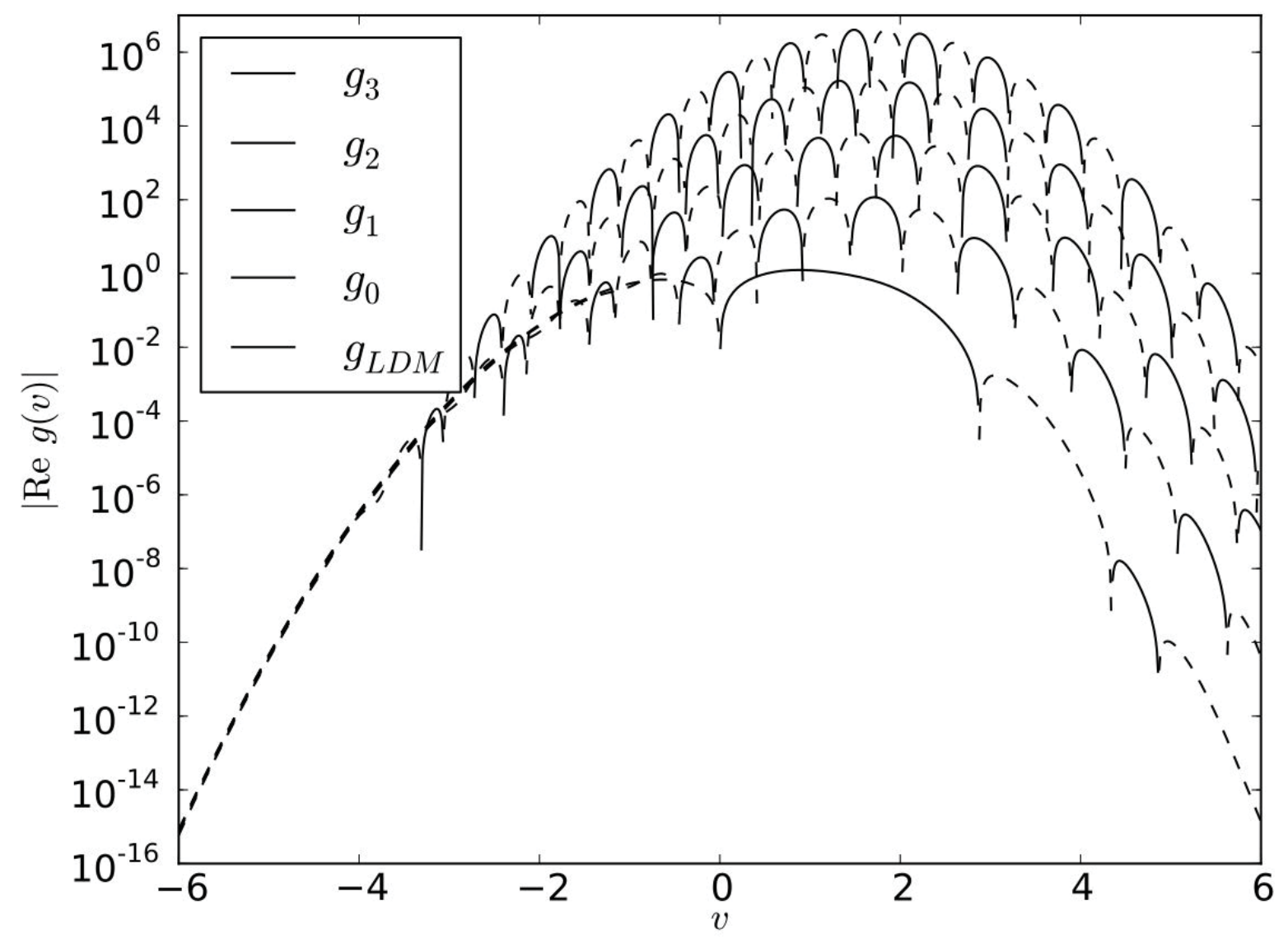

FIG. 9. Real part of the collisional eigenfunctions $g_{m}(v)$ shown on a semi-logarithmic scale. Solid lines indicate positive values, while dashed lines indicate negative values. Collisionality is $\mu=0.1$. Note that the maximum amplitude of the modes grows with mode number. In the plot here, the $g_{L D M}$ has the lowest maximum amplitude and $g_{3}$ has the largest.

\section{Decomposition into Eigenmodes}

Our goal in this work is to analyze simulation results by decomposing the plasma evolution into a linear combination of eigenmodes, which all evolve according to their eigenfrequencies, explaining the dynamics of the system as interference of those eigenmodes. In order to test our method, we have performed a simulation that starts with a superposition of 5 modes, all them equally weighted with $c_{m}=0.2$. Figure 10 should be compared to Fig. 8 , only that this time we perform a single simulation evolving all 5 modes at once, and instead of focusing on the electric field, we decompose the distribution function back into its eigenmode basis coefficients $c_{n}(t)$. We reproduce fundamentally the same behavior, as one would expect for a linear system, but with 


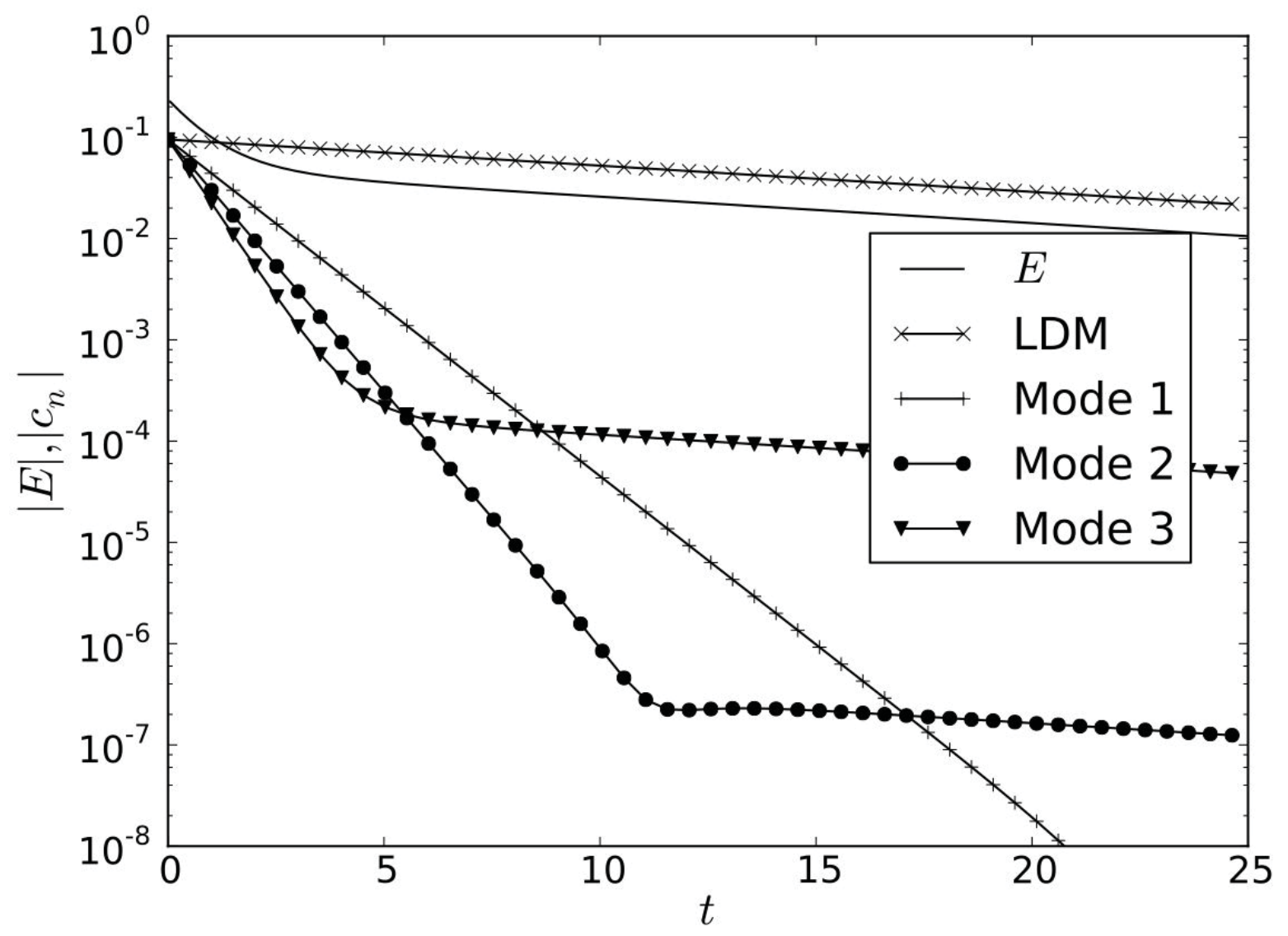

FIG. 10. Evolution of a superposition of LDM and Modes 1 to 4, initially weighted equally with coefficient 0.2 .

some caveats. LDM and modes 1 and 2 show the same evolution as before. Mode 3 turns over into the LDM behavior at an early point, while Mode 4 shows bleeding from other modes right away and is therefore not plotted. The main reason for the additional numerical difficulty lies in the fact that the numerical modes are not exactly orthogonal, as previously shown in Table III, which makes it impossible to obtain an exact decomposition. In addition, the eigenmodes and orthogonality relations are written for modes expanded of asymmetrically weighted Hermite functions, while the Kinetic Code uses symmetrically weighted polynomials, incurring additional conversions when setting up the initial conditions and analyzing distribution functions. In fact, the eigenmodes are so sensitive to small numerical errors that 64-bit floating point precision turned out to be insufficient to maintain even the already limited orthogonality under conversion between the bases. We have hence implemented the conversion routines in high-precision arithmetic using the mpmath Python 


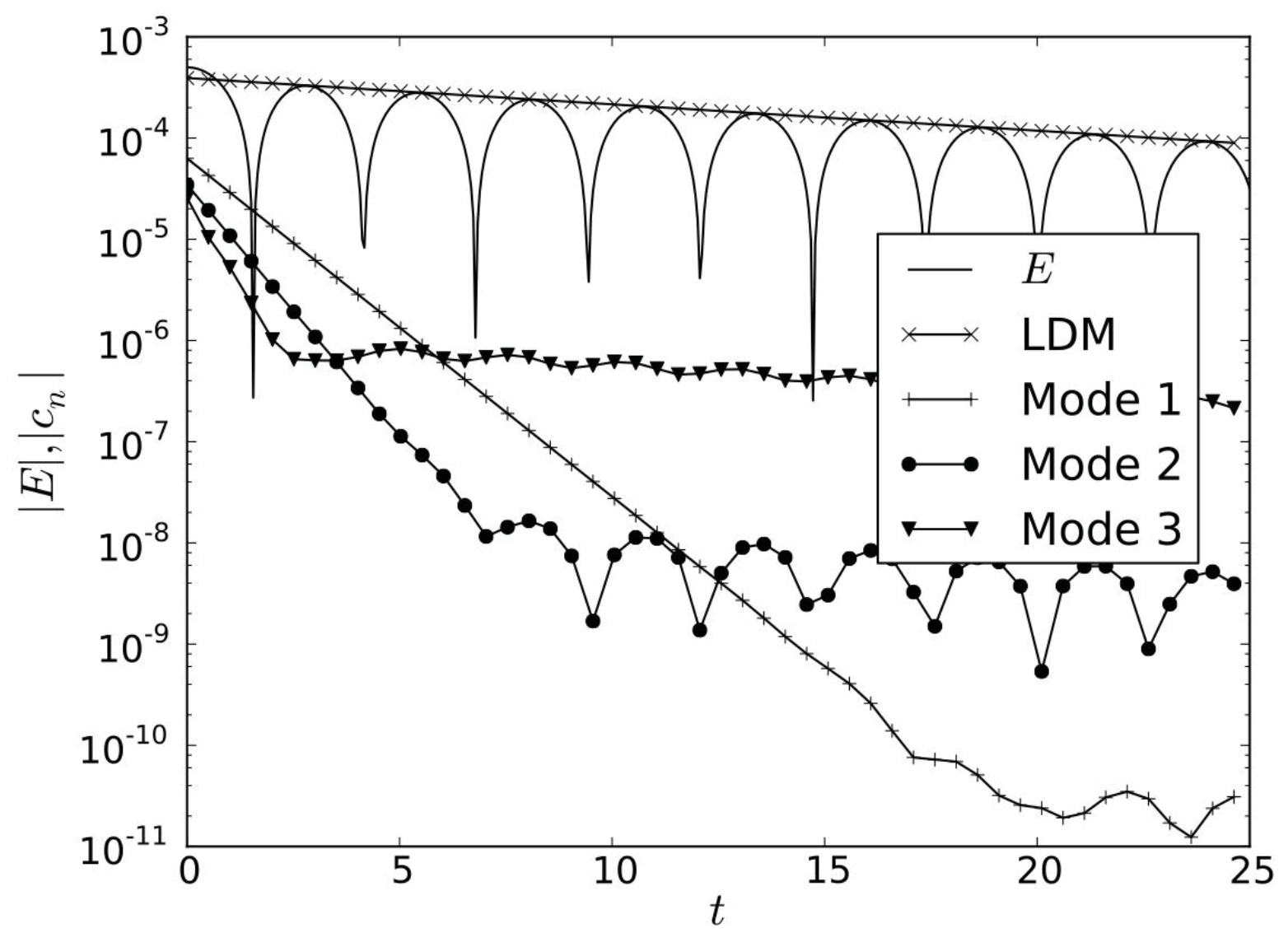

FIG. 11. Simulation initialized with a standing Langmuir wave, shown is the electric field $E$ and the magnitude of the decomposition coefficients $\left|c_{n}\right|$ vs time.

module ${ }^{17}$.

\section{Collisional Landau damping of a standing wave}

We now analyze the temporal evolution of a standing Langmuir wave in the presence of collisions. The electric field, as shown in Fig. 11 shows the expected standing wave oscillation, enveloped by exponential decay at the expected growth rate from the least damped mode. The composition of the initial Maxwellian into LDM and Modes 1 to 4 is also shown, and those components behave similarly to the test case where we used an equally weighted superposition of modes as initial condition. Modes 1 to 4, as expected, show rapid exponentially decay, leaving only the least damped mode to support the observed total electric field. The evolution, in fact, looks quite similar to our synthetic test initial condition, where we superposed 5 modes with equal 
weights, which subsequently decayed at their respective growth rates. In particular, the turning over of the fast exponential decay for the higher modes into slower decay at LDM rate is just the previously described numerical artifact. Our simulations also explain why the first "bump" in the electric field is higher than the exponential fit would have us expect, a phenomenon also observed in collisionless simulations, e.g. ${ }^{12}$. Landau damping describes the late time evolution of the electric field, however, there is an initial transient phase. In the context of the collisional system, the transient phase can be explained in terms of the additional modes which contribute to the initial condition. These modes also carry electric field, which explains the initially higher values, however, as they rapidly decay away, their contribution becomes insignificant quickly and the evolution of the electric field is well described by just the least damped mode itself.

\section{E. Completeness}

The NBS modes were shown to be a complete set of eigenmodes by ${ }^{10}$. First, we will examine the completeness problem by using numerically constructed linear eigenmodes from the method described at the end of the last section. Using this method, an eigenmode $g_{m}(v)$ is expanded in the form

$$
g_{m}(v)=\sum_{n=0}^{\infty} a_{m n} H_{n}(v) e^{-v^{2}},
$$

based on normalized variables. Numerically of course the summation over $n$ can only be done up to a finite number of modes number, say $n_{\max }$, since only a finite number of $a_{m n}$ can be calculated. In the same way, the adjoint function corresponding to $g_{m}(v)$ can be obtained,

$$
G_{m}(v)=\sum_{n=0}^{\infty} A_{m n} H_{n}(v) .
$$

The orthonormal condition requires

$$
\int_{-\infty}^{\infty} g_{m}(v) G_{n}(v) d v=\delta_{m n}
$$

or

$$
\sum_{l=0}^{\infty} a_{m l} A_{n l}=\delta_{m n} .
$$

In other words, the transpose matrix $\mathbf{A}^{\mathrm{T}}$ is the right inverse of the matrix a. Numerically this relationship can be confirmed to a high accuracy by including enough modes in the summations in Eqs. (34) and (35), i.e., with a large enough $n_{\max }$. 
Using the same formulation, the completeness condition then requires

$$
\sum_{m=0}^{\infty} g_{m}(v) G_{m}\left(v^{\prime}\right)=\delta\left(v-v^{\prime}\right),
$$

or

$$
\Gamma_{p q} \equiv \sum_{m=0}^{\infty} a_{m p} A_{m q}=\delta_{p q} .
$$

In other words, the transpose matrix $\mathbf{A}^{\mathrm{T}}$ is also the left inverse of the matrix $\mathbf{a}$, or that $\mathbf{a}$ is invertible. Again, numerically we can only sum up a finite number of terms in Eq. (39), or that $m$ can only take up to $m_{\max }$, the number of eigenmodes that can be evaluated accurately.

Unlike the orthonormal condition, Eq.(37), the completeness condition, Eq. (39), is much more difficult to test numerically. This is because the eigenmode $g_{m}(v)$ gets more and more singular in the $v$ space for larger $m$, and thus is more and more difficult to be expanded accurately in the form of Eq. (34) for a given finite number of terms, $n_{\max }$. In other words, the number of eigenmodes, $m_{\max }$, that can be calculated accurately is not large enough. As a matter of fact, this is much more so for the temporal problem than the spatial problem. When $m_{\max }$ is not too large, there are enough terms in the sum in Eq. (39) to get to converged values.

Facing this great difficulty, one way to proceed in order to at least testing the numerical framework, rather than studying physically interesting cases, is to consider the spatial problem when the collision is strong. This is because $g_{m}(v)$ is more singular, i.e., with very fine structures in the $v$ space with very small widths in the small $\mu$ limit. In the case of large $\mu$, the eigenmodes are smooth enough in the $v$ space that a large $m_{\max }$ of eigenmodes can be calculated accurately.

As an example, we show in Table V, some results of the calculation of $\Gamma_{p q}$ as defined in Eq. (39) for the first few values of $p$ and $q$, for the case with $\mu=1, \alpha=0.1, m_{\max }=5020, n_{\max }=$ 21600. We see that generally these values of $\Gamma_{p q}$ differ from the expected value of $\delta_{p q}$ in the order

of $O\left(10^{-2}\right)$.Note that $\Gamma_{p q}$ is identically zero by symmetry if $p$ and $q$ are not both even or both odd. The reason we cannot get even better agreement is again because of a finite $m_{\max }$ that can be calculated. In fact, in the calculation of each $\Gamma_{p q}$, the trend in convergence is not consistent with the theoretical expectation, although the rate of convergences appears to be quite slow.

\section{CONCLUSION}

In this paper, we report results from a kinetic Eulerian code in $1 \mathrm{~d}-1 \mathrm{v}$ space that includes the Lenard-Bernstein collision operator. Some standard tests of the code are given. We have shown 


$$
\begin{array}{lll}
p & q & \Gamma_{p q} \\
\hline 0 & 0 & 0.99999925+0.011 i \\
1 & 1 & 1.0000007-7.1 \times 10^{-7} i \\
0 & 2 & 0.016-0.008 i \\
2 & 0 & 1.1 \times 10^{-6}-0.008 i \\
2 & 2 & 0.989-0.0056 i \\
1 & 3 & -3.4 \times 10^{-6}-9.2 \times 10^{-7} i \\
3 & 1 & -1.5 \times 10^{-6}+2.6 \times 10^{-7} i \\
0 & 4 & -0.028+0.0069 i \\
4 & 0 & -2.6 \times 10^{-6}+0.0069 i \\
3 & 3 & 1.000003+3.9 \times 10^{-6} i \\
0 & 6 & 0.038-0.0063 i \\
6 & 0 & 5.0 \times 10^{-6}-0.0063 i \\
2 & 4 & 0.0195-0.0049 i \\
4 & 2 & 0.0098-0.0049 i \\
1 & 5 & 6.5 \times 10^{-6}+5.8 \times 10^{-6} i \\
5 & 1 & 2.3 \times 10^{-6}+3.9 \times 10^{-7} i
\end{array}
$$

TABLE V. Numerical Test of Completeness

Numerical values of $\Gamma_{p q}$ as defined in Eq. (39) for the first few values of $p$ and $q$, for the case with $\mu=1$,

$$
\alpha=0.1, m_{\max }=5020, n_{\max }=21600 .
$$

that a non-thermal distribution is thermalized by the LB operator. We have examined the effect that collisions have on numerical recursion. Significantly, we have benchmarked the code with the NBS modes.

We have discussed in detail our efforts in testing the orthogonality and completeness relation of NBS eigenmodes. The numerical challenges in providing definitive tests are quite formidable, but we have presented some evidence in support of these properties. 


\section{ACKNOWLEDGEMENT}

This work was conducted at the Center for Integrated Computation and Analysis of Reconnection and Turbulence (CICART) at the Institute for the Study of Earth, Oceans, and Space located at the University of New Hampshire. The research of C.B. is supported by an appointment to the NASA Postdoctoral Program at the Goddard Space Flight Center, administered by Oak Ridge Associated Universities through a contract with NASA. K.G. is supported by NSF grant CNS0855145 and DOE grant ER55093. C.S.N. is supported in part by a National Science Foundation Grant PHY-1004357, and by the National Science Foundation of China NSFC under Grant No. 41128004.

\section{REFERENCES}

${ }^{1}$ L. Landau. On the vibrations of an electronic plasma. Journal of Soviet Physics, 10(1):25-34, 1946.

${ }^{2}$ N. G. van Kampen. On the theory of stationary waves in plasmas. Physica, 21:949-963, 1955.

${ }^{3}$ K. M. Case. Plasma Oscillations. Annals of Physics, 7:349-364, 1959.

${ }^{4}$ A. Lenard and I.B. Bernstein. Plasma oscillations with diffusion in velocity space. Physical Review, 112(5):1456-1459, 1958.

${ }^{5}$ M. N. Rosenbluth, W. M. MacDonald, and D. L. Judd. Fokker-Planck Equation for an InverseSquare Force. Physical Review, 107:1-6, 1957.

${ }^{6}$ P. L. Bhatnagar, E. P. Gross, and M. Krook. A Model for Collision Processes in Gases. I. Small Amplitude Processes in Charged and Neutral One-Component Systems. Physical Review, 94:511-525, 1954.

${ }^{7}$ F. Skiff, S. De Souza-Machado, W.A. Noonan, A. Case, and T. N. Good. Linear kinetic modes in weakly collisional plasma. Physical Review Letters, 81(26):5820-5823, 1998.

${ }^{8}$ S. De Souza-Machado, M. Sarfaty, and F. Skiff. Kinetic modes in a hot magnetized and weakly collisional plasma. Physics of Plasma, 6(6), June 1999.

${ }^{9}$ C.S. Ng, A. Bhattacharjee, and F. Skiff. Kinetic eigenmodes and discrete spectrum of plasma oscillations in a weakly collisional plasma. Physical Review Letters, 83(10):1974-1977, 1999.

${ }^{10}$ C.S. Ng, A. Bhattacharjee, and F. Skiff. Complete spectrum of kinetic eigenmodes for plasma oscillations in a weakly collisional plasma. Physical Review Letters, 96(2), 2004. 
${ }^{11}$ R.W. Short and A. Simon. Damping of perturbations in weakly collisional plasmas. Physics of Plasmas, 9(8), 2002.

${ }^{12}$ J.W. Schumer and J.P. Holloway. Vlasov simulations using velocity-scaled hermite representations. Journal of Computational Physics, 144:626-661, 1998.

${ }^{13}$ F. C. Grant and M. R. Feix. Fourier-Hermite Solutions of the Vlasov Equations in the Linearized Limit. Physics of Fluids, 10:696-702, 1967.

${ }^{14}$ G. Joyce, G. Knorr, and H. K. Meier. Numerical Integration Methods of the Vlasov Equation. Journal of Computational Physics, 8:53, 1971.

${ }^{15}$ M. Shoucri and G. Knorr. Numerical Integration of the Vlasov Equation. Journal of Computational Physics, 14:84, 1974.

${ }^{16}$ A. Klimas. A method for overcoming the velocity space filamentation problem in collisionless plasma model solutions. Journal of Computational Physics, 68:202-226, 1987.

${ }^{17}$ Fredrik Johansson and others mpmath: a Python library for arbitrary-precision floating-point arithmetic (version 0.14), 2010. http: / / code.google.com/p/mpmath/. 